\title{
An Experimental Study of Photocatalytic Degradation of Congo Red Using Polymer Nanocomposite Films
}

\author{
Mashael Nasser Alshabanat $(\mathbb{D})$ and Murefah M. AL-Anazy \\ Chemistry Department, Science College, Princess Nourah Bint Abdulrahman University, Riyadh, Saudi Arabia \\ Correspondence should be addressed to Mashael Nasser Alshabanat; ma.naif@hotmail.com
}

Received 24 July 2018; Revised 13 October 2018; Accepted 22 October 2018; Published 2 December 2018

Academic Editor: Carola Esposito Corcione

Copyright (c) 2018 Mashael Nasser Alshabanat and Murefah M. AL-Anazy. This is an open access article distributed under the Creative Commons Attribution License, which permits unrestricted use, distribution, and reproduction in any medium, provided the original work is properly cited.

Eco-friendly polymer nanocomposite films were synthesized using biodegradable polymers of chitosan and polyvinyl alcohol as polymeric matrices and carbon black nanoparticles as the reinforcement. These films were applied to study their applicability to industrial wastewater purification as a photocatalyst for degradation of Congo red as a target pollutant and to study the effect of the polymeric matrix types of the films on their performance as a semiconductor photocatalyst. Fourier-transform infrared (FTIR) spectra and X-ray diffraction (XRD) were used to characterize the films. Visible light photocatalytic degradation of Congo red as a pollutant under various operational conditions of $\mathrm{pH}$, dye concentration, contact time, and light intensity was performed. Photocatalytic results revealed that the polymeric substrate type does not play a major role in the photodegradation of the dye, and the best operational conditions were at a $\mathrm{pH}$ of 6 and a dye solution concentration of $8 \mathrm{mg} / \mathrm{L}$.

\section{Introduction}

Dyes are extensively used in the industrial sector such as the textile industry, rubber, paper, leather, and cosmetics [1]. However, the discharge of dyes could lead to water pollution problems because of their toxicity.

Several techniques are used to remove such contaminants. These techniques can be categorized into conventional methods such as adsorption, coagulation, and flocculation; established methods such as ion exchange, membrane separation, and oxidation; and emerging methods such as biodegradation and microbial treatment [2].

Currently, the removal process based on photocatalysis is attracting great attention at the applied research level as a possible solution to the environmental pollution problem. The primary mechanism of this process involves the decomposition of organic contaminants into carbon dioxide and water by utilizing light energy. Semiconductor photocatalysts absorb the light energy, thus initiating the oxidation reactions by generating hole $\left(\mathrm{h}^{+}\right)$and electron $\left(\mathrm{e}^{-}\right)$pairs, which can generate free radicals such as hydroxyl $(\bullet \mathrm{OH})$ radicals [3]. These free radicals work as oxidizers of organic pollutants ([3-5] cited in [3]). Therefore, the photocatalytic oxidation reaction requires a source of light with energy in the visible or UV spectrum, or solar energy. However, to generate the $\mathrm{h}^{+}$and $\mathrm{e}^{-}$pairs, a semiconductor catalyst must be capable of absorbing light energy equal to or greater than its bandgap energy ([3-5] cited in [3]).

Currently, there is a tendency to use semiconductor polymer nanocomposite systems as photocatalysts. Several researchers have published works based on this approach [6-10]. For example, and in previous study, ZnO/PMMA nanocomposite as a photocatalyst had been prepared, and the results demonstrated that the proposed nanocomposite is a promising candidate for water treatment [6]. The photocatalytic activity of $\mathrm{TiO}_{2} /$ chitosan (nTC) nanocomposites had also been prepared in another study using hydrothermal process, and the results showed that the best performance of the nanocomposites was with the amount of nTC of $0.25 \mathrm{~g}$ and irradiation time of $120 \mathrm{~min}$ [7]. A photocatalytic degradation of metronidazole and methylene blue by PVAassisted $\mathrm{Bi}_{2} \mathrm{WO}_{6}$-CdS nanocomposite film had been studied under visible light irradiation by Rajendran et al. [8], and in 
another work [9], a crosslinked-chitosan/nano-CdS (CS/nCdS) composite photocatalyst had been prepared and characterized and a photocatalytic activity under visible light irradiation was studied under various conditions of catalyst amount, $\mathrm{pH}$, and concentration of target dye. A review recently published provided comprehensive information on polymer nanocomposites in the field of water purification [2]. According to this review, the features of such materials for water purification is their stability, low cost, and improved processability [2]; in addition, the technique eliminates the need for the recovery of the particles after the treatment process [6].

Polymer nanocomposites consist of reinforcement filler nanoparticles embedded and incorporated into the polymeric substrate, thereby providing a combination of great properties of the produced film. The polymeric matrix improves the processability of a nonpolymeric constituent [2]; addition of conductive fillers into an insulating polymeric matrix can provide the film with conductive properties.

This study aims to prepare an eco-friendly semiconductor polymer nanocomposite film as a photocatalyst for the degradation of wastewater pollutants and to study the influence of different polymeric matrix types of the nanocomposite thin films on their performance as photocatalysts, considering the effect of different effective factors including the concentration of the dye, $\mathrm{pH}$, light intensity, and contact time. To achieve this goal, two target polymers were used in this study. The first is chitosan because it is biocompatible, is biodegradable, has multiple functional groups, and is nontoxic and cheap [11, 12]. The second is polyvinyl alcohol (PVA) because it is also completely biodegradable and has excellent physical properties and chemical resistance [13].

\section{Experimental}

2.1. Materials. Congo red (CR) (Aldrich-Sigma) was used as a model pollutant dye. It has the chemical formula $\mathrm{C}_{32} \mathrm{H}_{22} \mathrm{~N}_{6} \mathrm{Na}_{2} \mathrm{O}_{6} \mathrm{~S}_{2}$; i.e., it is a sodium salt of benzidinediazobis-1-naphthylamine-4-sulfonic acid [14].

Two types of biodegradable polymers were used as previously indicated. They included chitosan with molecular weight of 100,000-300,000 (Acros Organics), PVA with degree of polymerization of 1700-1800, hydrolysis (mole\%) of 98-99, volatiles of max 5\%, and ash of max $0.7 \%$ (Loba Chemie). The reinforcement material of the nanocomposite films is furnace carbon black (Degussa) with a particle size of $95 \mathrm{~nm}$.

\subsection{Methods}

2.2.1. Preparation of Nanocomposite Films. To prepare the polymer nanocomposite film, the nanoparticle carbon materials were spread out over the polymer matrix in a solution form, and the solution was stirred magnetically under a suitable temperature for $3 \mathrm{~h}$. Next, the mixture was cast into a Petri dish and left at ambient temperature to dry, and the solvent was allowed to evaporate completely (Figure 1).

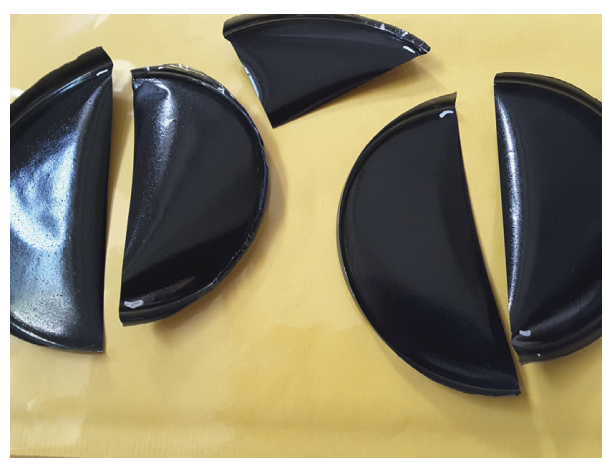

Figure 1: A photograph of the prepared films.

To prepare the polymer solutions, $2 \mathrm{~g}$ of chitosan was dissolved in $100 \mathrm{~mL}$ of $2 \%$ acetic acid and stirred magnetically until a homogenous solution was obtained, while $2 \mathrm{~g}$ of PVA was dissolved in $100 \mathrm{~mL}$ of distilled water and stirred magnetically at $90^{\circ} \mathrm{C}$ until a homogenous solution was obtained. The samples were labeled as Chit/C and PVA/C for the chitosan nanocomposites and PVA nanocomposite film photocatalysts, respectively. They were characterized spectroscopically by recording FT-IR spectra using Fouriertransform infrared (FT-IR) spectroscopy 1000, PerkinElmer ranging $4000-400 \mathrm{~cm}^{-1}$, and the X-ray diffraction using PRO X-ray diffractometer made in Holland.

2.2.2. Photodegradation Studies. In general, photodegradation studies were performed via a fixed surface area $(1.5 \mathrm{~cm} \times 1.5 \mathrm{~cm})$ of the films, after first placing them in a $250 \mathrm{~mL}$ Erlenmeyer flask. Then, $50 \mathrm{~mL}$ of the standard dye solution was added to the flask and stirred under dark conditions in a photocatalytic unit for $30 \mathrm{~min}$ to ensure an adsorption-desorption equilibrium is attained. A highpressure mercury lamp (Philips) of $20-120 \mathrm{~W}$ emitting visible light irradiation was placed above the dye solution at a fixed distance of $20 \mathrm{~cm}$. The solution was stirred continuously, using a magnetic stirrer (model Thermo Cimarec) at $25^{\circ} \mathrm{C}$. The absorption of the samples was measured at $\lambda=$ $502 \mathrm{~nm}$ (using UV-Vis spectrophotometer Jenway-6800, UK) (Figure 2). The degradation yield can be defined as follows [15]:

$$
\text { degradation yield }(\%)=\frac{\left(C_{0}-C_{e}\right)}{C_{0}} \times 100,
$$

where $C_{0}$ is the initial dye concentration and $C_{\mathrm{e}}$ is the concentration of dye after photodegradation $(\mathrm{mg} / \mathrm{L})$.

To study the effect of $\mathrm{pH}$, a series of solutions with a constant concentration of $8 \mathrm{mg} / \mathrm{L}$ of the dye with a $\mathrm{pH}$ ranging from 4 to 8 using Hydrolab (WTW multi 340i set, Germany) were used. The solutions were placed in a photocatalytic unit at room temperature $\left(25^{\circ} \mathrm{C}\right)$ for a constant contact time of $120 \mathrm{~min}$; the light intensity was $525 \mathrm{~W} / \mathrm{m}^{2}$. The absorbance of the solution before and after the irradiation was measured using a spectrophotometer.

To study the effect of the dye concentration, and at $25^{\circ} \mathrm{C}$, about $50 \mathrm{~mL}$ of the solution with different dye concentrations of $2,4,6,8,10$, and $20 \mathrm{mg} / \mathrm{L}$ at a fixed $\mathrm{pH}$ of 6 was used, and 


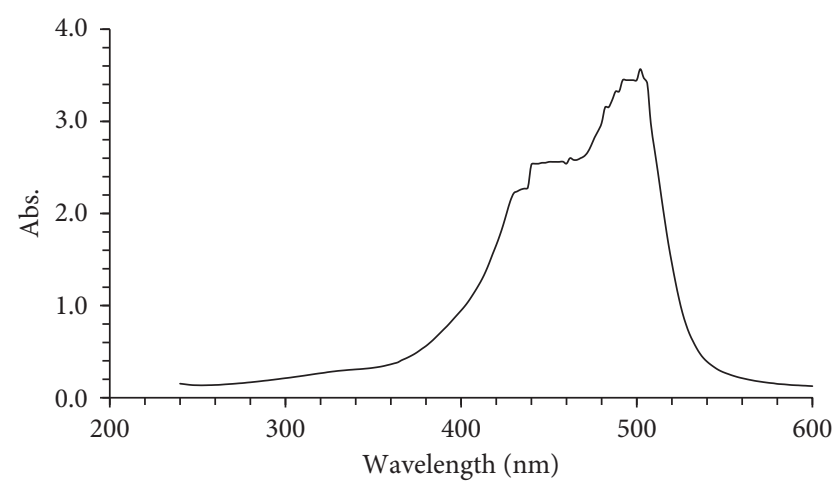

Figure 2: UV-Vis absorption spectrum of CR.

then, the catalyst was added. Before irradiation, the system was magnetically stirred for 30 min under dark conditions to establish the appropriate adsorption-desorption equilibrium between the catalytic surface and the dye. The irradiation time was limited to $120 \mathrm{~min}$ with a light intensity of $525 \mathrm{~W} / \mathrm{m}^{2}$. The absorbance of the solution before and after the irradiation was measured using the spectrophotometer.

To investigate the effect of contact time, a constant concentration of $8 \mathrm{mg} / \mathrm{L}$ of the dye with $\mathrm{pH}$ adjusted to 6 was placed in the photocatalytic unit at room temperature $\left(25^{\circ} \mathrm{C}\right)$. This was done for different contact times ranging from 15 to $180 \mathrm{~min}$ with the light intensity set at $525 \mathrm{~W} / \mathrm{m}^{2}$. The absorbance of the solution before and after the irradiation was measured using a spectrophotometer.

Finally, the effect of light intensity was studied using approximately $50 \mathrm{~mL}$ of solution with a fixed dye concentration of $8 \mathrm{mg} / \mathrm{L}$ at $25^{\circ} \mathrm{C}$ and a fixed $\mathrm{pH}$ of 6 , followed by the addition of a catalyst. Before irradiation, the system was magnetically stirred for $30 \mathrm{~min}$ under dark conditions to establish the appropriate adsorption-desorption equilibrium between the catalytic surface and the dye. The irradiation time was limited to $120 \mathrm{~min}$ with different light intensity values including $20,40,60$, and $80 \mathrm{~W}(175,350,525$, and $700 \mathrm{~W} / \mathrm{m}^{2}$, respectively) using different mercury lamps; each lamp had a power rating of $20 \mathrm{~W}$ and emitted a light intensity of $175 \mathrm{~W} / \mathrm{m}^{2}$. The absorbance of the solution before and after the irradiation was measured using a spectrophotometer.

\section{Results and Discussion}

3.1. Characterization. Figure 3 shows the FT-IR spectra of the polymers and the prepared nanocomposite films, and Table 1 summarizes their spectral data. The IR characteristics of the original chitosan are 3448, 2925, 2369, 1655, 1405 , and $1077 \mathrm{~cm}^{-1}$, assigned to $\mathrm{O}-\mathrm{H}$ stretching overlapped with $\mathrm{N}-\mathrm{H}$ stretching, aliphatic $\mathrm{C}-\mathrm{H}$ stretching, in plane $\mathrm{N}-\mathrm{H}$ bending, $\mathrm{C}-\mathrm{O}$ stretching vibration of primary alcoholic group [16, 17], and stretching vibration of hydroxy group ([18, 19] cited in [17]), respectively. These values are shifted to $3425,2924,2370,1546,1406$, and $1069 \mathrm{~cm}^{-1}$ of the Chit/C film, whereas the IR characteristics of the original PVA are 3404, 2926 and 2369, 1656, 1433, 1095 , and $670 \mathrm{~cm}^{-1}$ assigned to $\mathrm{O}-\mathrm{H}$ stretching, $\mathrm{CH}_{2}$ asymmetric and symmetric stretching, C-O stretching, C-H bending, and C-H outside the plan bond, respectively [20], shifted to $3400,2935,2370,1437,1092$, and $669 \mathrm{~cm}^{-1}$, respectively, of the film of PVA/C owing to incorporation of the carbon particles with the polymers.

The XRD pattern of Chit/C, PVC/C, and the original carbon black is shown in Figure 4 . The pattern of carbon black shows two broad peaks around $25^{\circ}$ and $43^{\circ}$ assigned to its amorphous structure [21]. As for the Chit/C film, there are three sharp peaks around $2 \theta=10.0^{\circ}, 12.0^{\circ}$, and $20.0^{\circ}$ and three broad peaks around $24^{\circ}, 32^{\circ}$, and $43^{\circ}$. The sharp beaks at $10.0^{\circ}$ and $20.0^{\circ}$ are assigned to crystal morphology of chitosan [22], whereas the broad and weak peaks at $24^{\circ}$ and $43^{\circ}$ are assigned to residual of the carbon black, and the other peaks at $12.0^{\circ}$ and $32^{\circ}$ are assigned to the formation of nanocomposites. As for the PVA/C, the pattern revealed an amorphous form with three board peaks around $20^{\circ}$ and $30^{\circ}$, assigned to the nanocomposite film, and the peak around $40^{\circ}$ is assigned to the carbon black, remaining in the nanocomposite. The residual of the carbon black in the XRD pattern of the nanocomposite films of Chit/C and $\mathrm{PVA} / \mathrm{C}$ suggests that the attraction with the polymer was partial.

3.2. Photodegradation Studies. To study the effect of the various parameters on the degradation process, the mechanism of the process should be understood. Overall, the suggested mechanism for the photodegradation process of CR dye is as follows $[23,24]$.

Firstly, the molecules of the CR dye are adsorbed on the photocatalyst surface, which is then irradiated by visible light, leading to the excitation of electrons in the valance band to the conduction band leaving behind positive holes $\left(\mathrm{h}^{+}\right)$in the valance band. Subsequently, on the surface of the photocatalyst, the pair of $\mathrm{h}^{+}$and $\mathrm{e}^{-}$will react with the adsorbed water molecules, dissolved oxygen, and hydroxyl groups of the surface, producing free radicals of hydroxyl and superoxide radicals. The $\mathrm{h}^{+}$then reacts with water molecules to produce ${ }^{\bullet} \mathrm{OH}$ radicals, and the $\mathrm{e}^{-}$reduces the dissolved oxygen to superoxide anion radical $\mathrm{O}_{2}{ }^{\bullet-}$. These photogenerated radicals would degrade the dye molecules, forming intermediate products that completely break into $\mathrm{CO}_{2}, \mathrm{H}_{2} \mathrm{O}$, and ions of $\mathrm{NO}_{3}{ }^{-}$and $\mathrm{NH}_{4}{ }^{+}$as expressed in the following equations:

The first step (Equation (2)) [23, 24]:

$$
\begin{aligned}
& \text { Chit./C } \left.+\mathrm{h} v \longrightarrow \text { Chit./C( } \mathrm{h}_{(\mathrm{VB})}{ }^{+}+\mathrm{e}_{(\mathrm{CB})}{ }^{-}\right) \text {or } \\
& \text { PVA } / \mathrm{C}+\mathrm{h} v \longrightarrow \text { PVA/C }\left(\mathrm{h}_{(\mathrm{VB})}{ }^{+}+\mathrm{e}_{(\mathrm{CB})}{ }^{-}\right)
\end{aligned}
$$

The second step (Equations (3) and (4)) [23, 24]:

$$
\begin{aligned}
\text { Chit./C }\left(\mathrm{h}^{+}\right) \text {or PVA/C }\left(\mathrm{h}^{+}\right)+\mathrm{H}_{2} \mathrm{O} \longrightarrow & \mathrm{H}^{+}+{ }^{\bullet} \mathrm{OH} \\
\text { Chit./C }\left(\mathrm{e}^{-}\right) \text {or PVA/C }\left(\mathrm{e}^{-}\right)+\mathrm{O}_{2} \longrightarrow & \text { Chit./C or PVA/C } \\
& +\mathrm{O}_{2}{ }^{--}
\end{aligned}
$$

The third step (Equation (5)) [24]: 


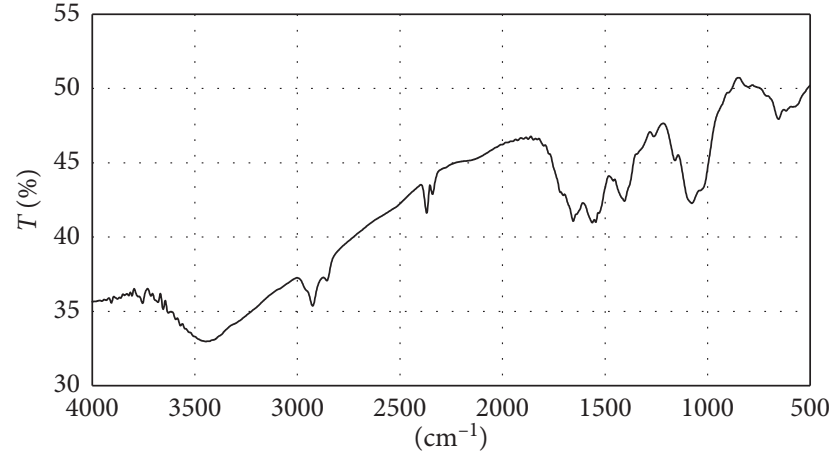

(a)



(c)

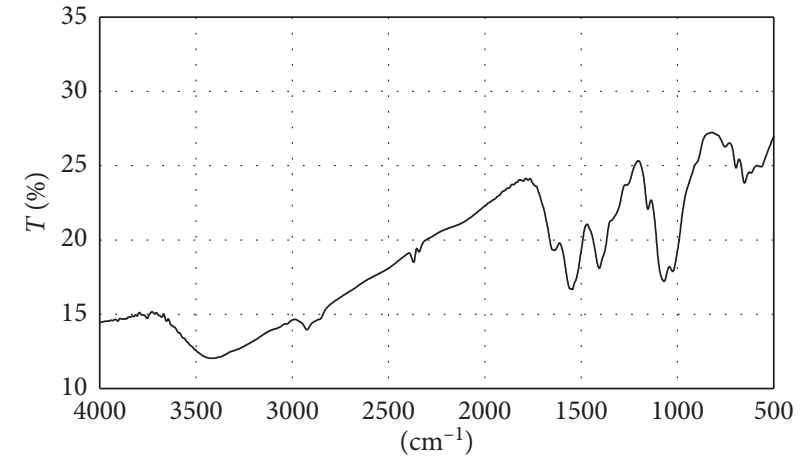

(b)

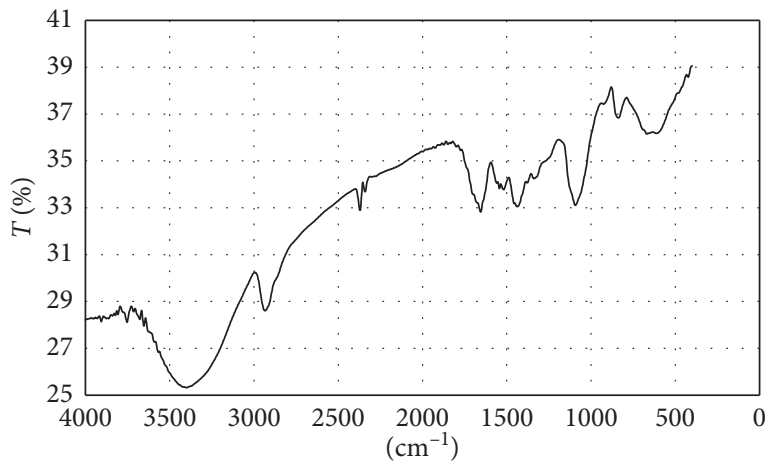

(d)

Figure 3: FT-IR spectra of chitosan, PVA, and the prepared films. (a) Chit, (b) Chit/C, (c) PVA, and (d) PVA/C.

TABLE 1: Spectral data of chitosan, PVA, and the prepared nanocomposite films.

\begin{tabular}{cccccc}
\hline $\begin{array}{l}\text { Chitosan } \\
\text { Position }\left(\mathrm{cm}^{-1}\right)\end{array}$ & Interpretation & $\begin{array}{l}\text { PVA } \\
\text { Position }\left(\mathrm{cm}^{-1}\right)\end{array}$ & $\begin{array}{c}\text { PVA/C } \\
\text { Interpretation }\end{array}$ \\
\hline 3448 & 3425 & O-H stretching overlapped with N-H stretching & 3404 & 3400 & $\begin{array}{c}\text { O-H stretching } \\
2925\end{array}$ \\
2369 & 2924 & Aliphatic C-H stretching & 2926 & 2935 & $\mathrm{CH}_{2}$ asymmetric and symmetric \\
1655 & 1546 & In plane N-H bending & 2369 & 2370 & stretching \\
1405 & 1406 & C-O stretching vibration of primary alcoholic group & 1433 & 1437 & C-H bending \\
1077 & 1069 & Stretching vibration of hydroxy group & 670 & 1092 & C-O stretching \\
\hline
\end{tabular}

CR molecules $+{ }^{\bullet} \mathrm{OH}+\mathrm{O}_{2}{ }^{\bullet-} \longrightarrow$ intermediate compounds

$$
\begin{aligned}
\longrightarrow & \mathrm{CO}_{2}+\mathrm{H}_{2} \mathrm{O}+\mathrm{NO}_{3}^{-} \\
& +\mathrm{NH}_{4}{ }^{+}
\end{aligned}
$$

In general, the electrical double layer of the solution/ solid interface is modified by $\mathrm{pH}$, affecting the adsorptiondesorption process, subsequently, releasing the photogenerated pairs $\mathrm{e}^{-}-\mathrm{h}^{+}$on the surface of the photocatalyst ([3, 25] cited in [3]). The absorption process mechanism under the effect of $\mathrm{pH}$ could be proposed by electrostatic interaction between the CR molecules and the active sites of the semiconductor surface $([26,27]$ cited in [25]), possibly affecting the encounter of the resulting molecules with ${ }^{\circ} \mathrm{OH}$ [26]. Depending on the prevailing forces whether attraction or repulsion, the overall reaction will either improve or restrain ([28] cited in [25]). Thus, $\mathrm{pH}$ plays an important role in the photodegradation of the CR dye.
Figure 5 illustrates the effect of $\mathrm{pH}$ on the photocatalytic degradation of CR by both the photocatalyst films. Obviously, the both catalysts mostly follow the same pattern towards the degradation process, wherein the percentage degradation increases with increasing $\mathrm{pH}$ until it reaches to 6 then decreases with further increasing in $\mathrm{pH}$. This observation suggests that the strongest electrostatic attraction force between the dye molecules and the surfaces of catalysts is at this value of $\mathrm{pH}$, leading to maximum activity of oxidation, and thereby, high photodegradation efficiency of the $\mathrm{CR}$ dye is obtained. The higher adsorption rate obtained at 6 of $\mathrm{pH}$ could be explained by the nature of the CR dye itself. The molecule CR dye is a dipolar [1], and it was found that, the color of CR dye solution is changeable from red to dark blue with decreasing $\mathrm{pH}$, suggesting that the ionic character nature of CR dye molecules depend on the medium $\mathrm{pH}$ [1]. At the acidic medium, the dye exists as a cationic form and as an anionic form at the basic medium [1]. When the medium of the solution is acidic, the cationic molecules of the CR dye 


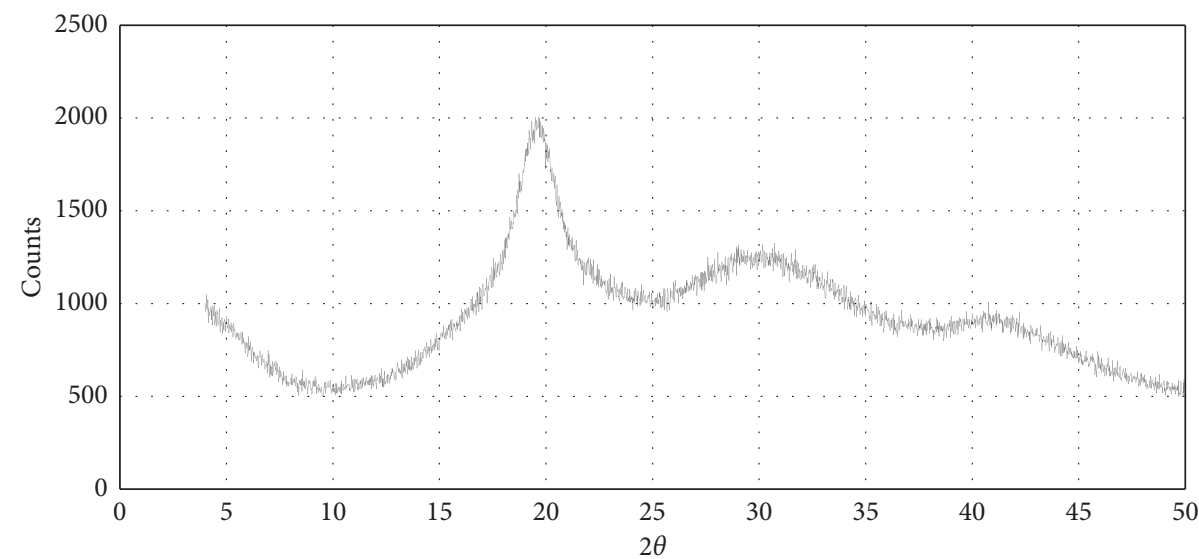

(a)

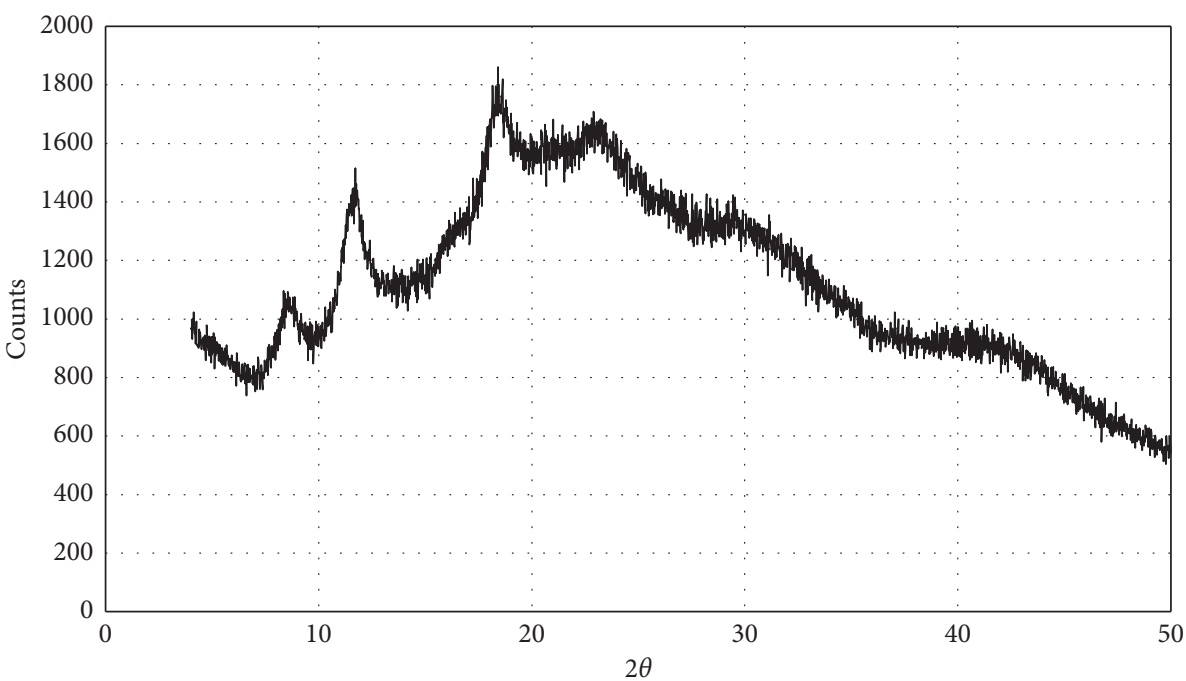

(b)

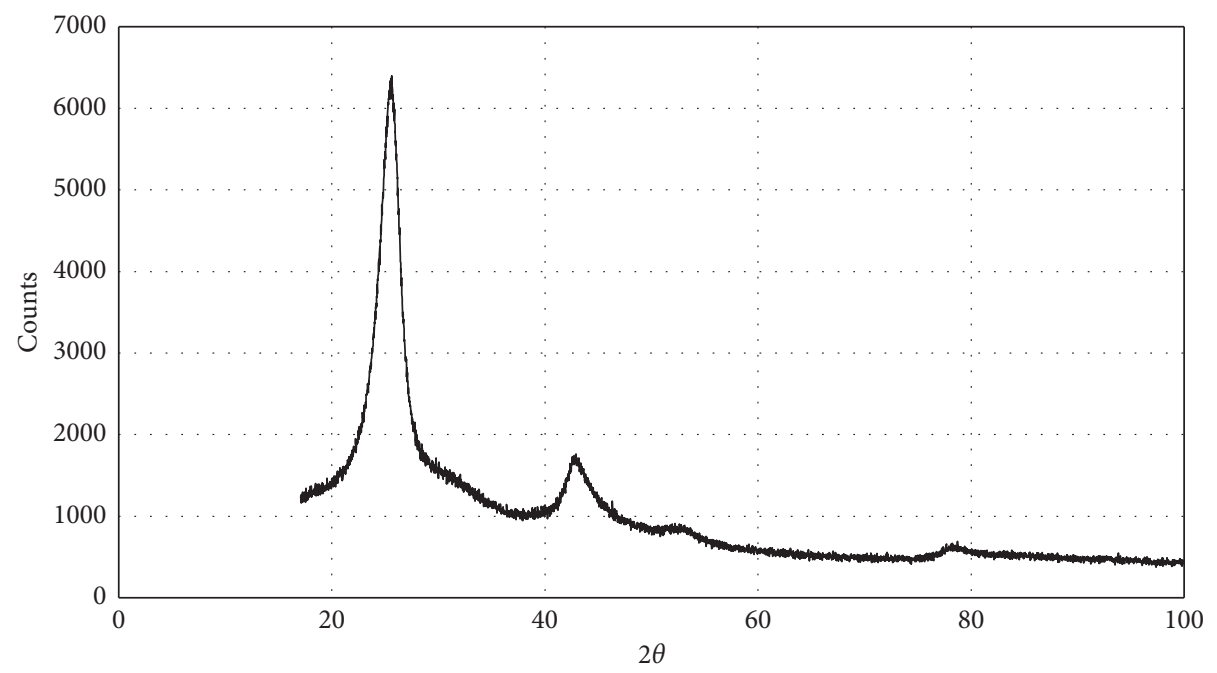

(c)

FIGURE 4: XRD patterns of carbon black and the prepared films. (a) PVA/C, (b) Chit/C, and (c) carbon black.

are repulsed with protons ions, resulting in a reduction of the adsorption process. However, the percentage decreases beyond this $\mathrm{pH}$ value because more hydroxyl ions are available in the alkaline medium, resulting in repulsion between the negative speciation of the dye molecules and the negatively charged hydroxyl ions.

The mechanism of the adsorption of the CR dye on the Chit/C surface at various $\mathrm{pH}$ values could be explained by 


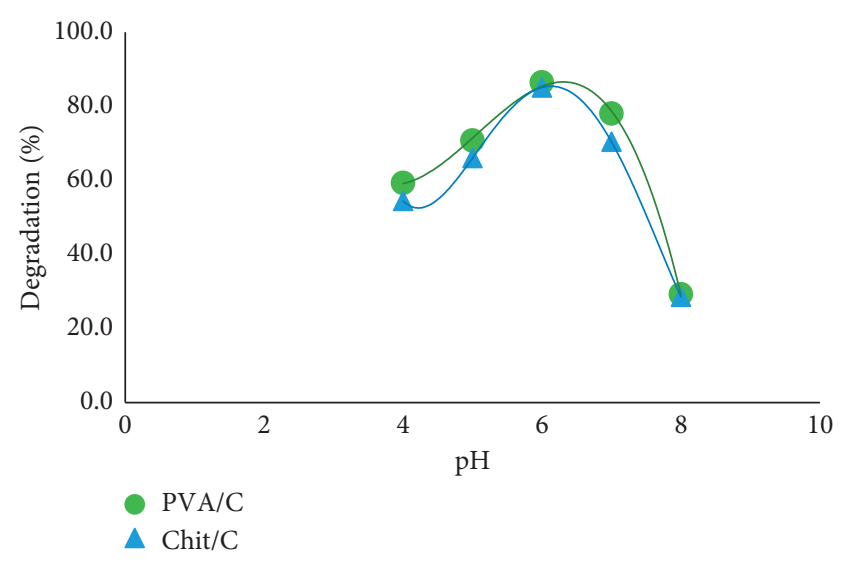

FIgure 5: Percentage photodegradation as a function of $\mathrm{pH}$.

the electrostatic interaction between the molecules of the dye and Chit/C. In the Chit/C photocatalyst, chitosan contains primary amino groups $\left(-\mathrm{NH}_{2}\right)$ with the hydroxyl group, making it a highly reactive polysaccharide [7, 9, 29]. In the acidic solution, the reactive $-\mathrm{NH}_{2}$ is easy to protonate to the $-\mathrm{NH}^{3+}$ form, expecting to contribute to the interaction with the ionic CR dye [29]. As mentioned above, CR exists as a cationic form at acidic $\mathrm{pH}$. Therefore, when a solution medium is acidic, the cationic molecules of CR dye are repulsed with the $\mathrm{H}^{+}$ions and $\mathrm{NH}_{3}{ }^{+}$ions, resulting in a reduction of the adsorption process on the Chit/C surface. Meanwhile, the adsorption of the CR dye on the PVA/C surface mechanism can be explained as follows.

For an acidic medium, the PVA surface is protonated as shown in equation (6) [30], leading to repulsion between the positive charges of the polymer surface and the dye, whereas in an alkaline medium, deprotonation occurs by the release of a free oxygen on the surface of the PVA [30], also leading to electrostatic repulsion as expressed in equation (7) [30]:

$$
\begin{gathered}
\mathrm{OH}^{-}+\mathrm{H}^{+} \longrightarrow \mathrm{OH}_{2}+\mathrm{OH}_{2}^{+}+\mathrm{CR}^{+} \longrightarrow \mathrm{OH}_{2}{ }^{+} \mathrm{CR}^{+} \\
\mathrm{OH}^{-} \longrightarrow \mathrm{O}^{-}+\mathrm{H}^{+} \mathrm{O}^{-}+\mathrm{CR}^{+} \longrightarrow \mathrm{O}^{-} \mathrm{CR}^{-}
\end{gathered}
$$

Figure 6 shows the effect of the initial dye concentrations on the percentage photodegradation by Chit/C and PVA/C catalysts. They almost follow the same pattern. The percentage degradation of the CR dye increases initially with an increase of the concentration until $8 \mathrm{mg} / \mathrm{L}$, because of the availability of the adsorption sites on the photocatalyst surface that enhances the ability of adsorption of the CR molecules. These sites are saturated with time as they get covered with the adsorbed molecules, causing a decline in the percentage of photodegradation with a further increase in the concentration.

A similar observation was reported elsewhere [7], which was explained by the increase in the dye concentration that leads to the increase in the dye adsorption on the active sites of the catalyst surface; hence, the competitive adsorption of oxygen on these sites decreases, which leads to a lower formation rate of $\mathrm{O}_{2}-\bullet$ and $\bullet \mathrm{OH}$ radicals. The study [7] suggested an explanation based on

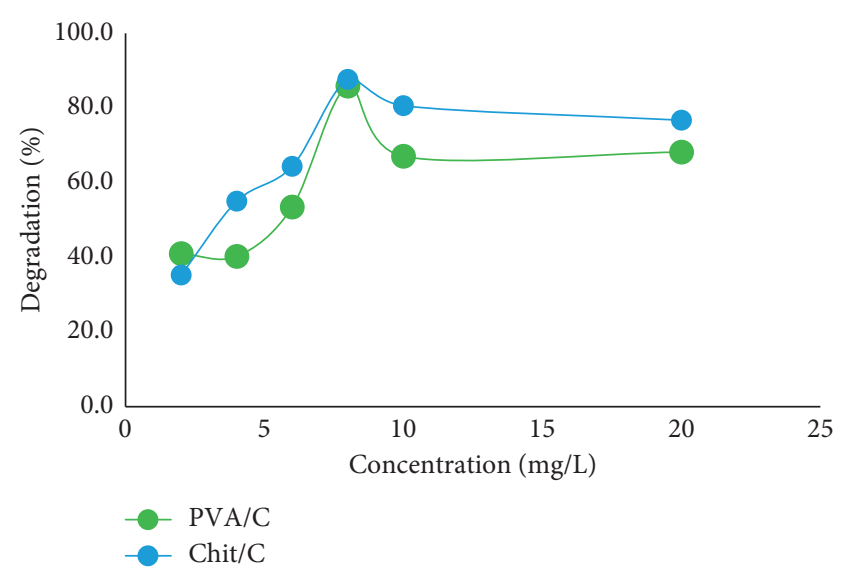

FIgURE 6: Percentage photodegradation as a function of initial concentration of CR dye.

the Beer-Lambert law, wherein the increase in the initial dye concentration decreases the path length of the photons entering the solution, resulting in a lower adsorption for the photon on the catalyst particles, which causes the photodegradation rate to be lower. Another suggested factor proposed by another study [7] is the competition for the photogenerated holes between the adsorbed molecules of water and the CR dye.

Figure 7 shows the effect of contact time on the photodegradation process for both photocatalysts. Clearly, the behavior of both catalysts seems to be similar. The percentage degradation increases rapidly in the first hour of irradiation exposure because of the enhancement of the excitation process of many electrons from the valence to conduction bands of the semiconductor photocatalysts, generating the pairs $\mathrm{e}^{-}-\mathrm{h}^{+}$in the photocatalyst surface, which are powerful for generating free radical oxidizers. Thereafter, there is a plateau that may result from the consumption of the catalyst.

Figure 8 shows the effect of light intensity on the degradation process. As shown, the patterns of the photodegradation by both the catalysts were initially the same, wherein it linearly increased, after which there was a decrease for the PVA/C photocatalyst, whereas Chit/C continued to increase slightly. The maximum percentage degradation values were $88.4 \%$ and $84.2 \%$ for Chit/C and $\mathrm{PVA} / \mathrm{C}$, respectively.

The difference in the behavior and associated values could be attributed to the difference in the photodegradation mechanism. The increase in the photodegradation process with an increase in the light intensity occurred because of the increase in the formation of the pair of $\mathrm{e}^{-}-\mathrm{h}^{+}$and the decrease in the percentage photodegradation for the PVA/C because of recombination of $\mathrm{e}^{-}-\mathrm{h}^{+}$.

From the above discussion, it is clear that Chit/C and PVA/C mostly behave the same, wherein they both showed a similar trend towards the photodegradation of CR, and the maximum values of percentage photodegradation were very close, as tabulated in Table 2, suggesting that the effect of polymeric substrate in our study could be neglected. This is possibly because the adsorbing CR molecules on the 


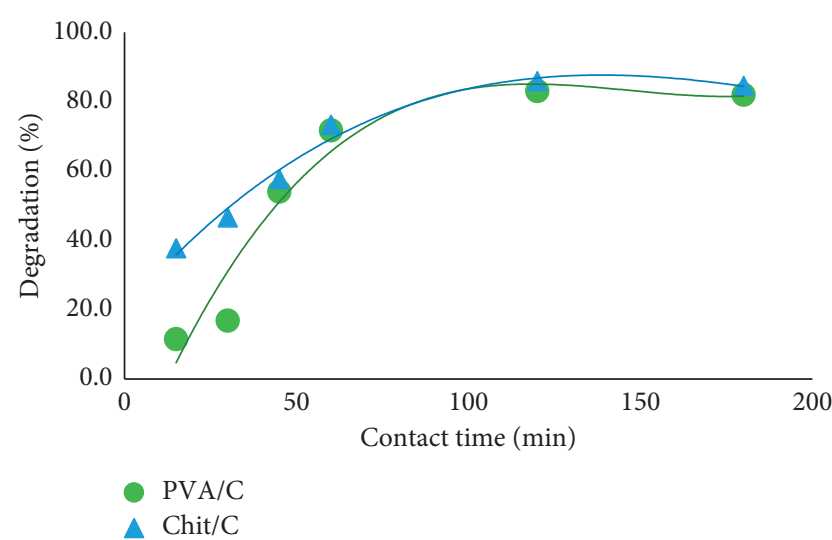

Figure 7: Percentage photodegradation as a function of contact time.

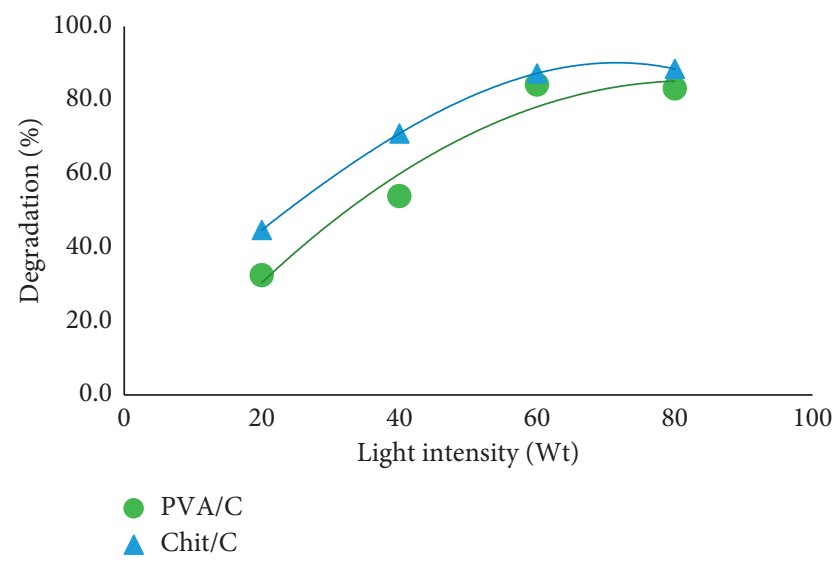

Figure 8: Percentage photodegradation as a function of light intensity.

TABle 2: Maximum percentage photodegradation (\%) of the CR dye by the prepared films with various parameters.

\begin{tabular}{lcccc}
\hline \multirow{2}{*}{$\begin{array}{l}\text { The } \\
\text { films }\end{array}$} & $\mathrm{pH}$ & $\begin{array}{c}\text { Initial } \\
\text { concentration }\end{array}$ & $\begin{array}{c}\text { Contact } \\
\text { time }\end{array}$ & $\begin{array}{c}\text { Light } \\
\text { intensity }\end{array}$ \\
\hline Chit/C & 85.1 & 87.6 & 85.9 & 88.4 \\
PVA/C & 86.4 & 86 & 83 & 84.2 \\
\hline
\end{tabular}

photocatalyst surface, which presents a first step in the photodegradation mechanism, occurred through the same bonding forces such as the combination of Van der Waals, hydrogen bonding, and/or of other forces by electrostatic interaction between the CR dye molecules and protonated and deprotonated groups of the polymeric substrate in nanocomposite films. Meanwhile, in other steps of the photocatalysis mechanism, the conductive property of the films is a key factor, wherein the polymeric matrix itself is insolated, and this property is given by the carbon black particles. The presence of carbon black particles lowers the overall resistivity by several orders of magnitude, meaning that the transition of the nanocomposite from insulating to conducting is a function of carbon black concentration, which was fixed in both catalysts. Therefore, in our study, the polymeric substrate, chitosan, and PVA did not play a big role in the photocatalytic degradation of the CR dye.

\section{Conclusion}

Chitosan/carbon black and polyvinyl alcohol/carbon black nanocomposite films were prepared and characterized using Fourier-transform infrared (FT-IR) spectra and X-ray diffraction (XRD) techniques. Visible light was used as a source of light for the photocatalytic degradation process under various operational conditions of $\mathrm{pH}$, dye concentration, contact time, and light intensity. The findings showed that the role of the polymeric matrix on the photodegradation of the dye was not very impressive. Furthermore, the photodegradation process improves as the light intensity was high and on increasing the contact time. The best operational conditions of the $\mathrm{pH}$ and concentration dye solution were at 6 and $8 \mathrm{mg} / \mathrm{L}$, respectively. The results showed that these films could be good materials as photocatalysts and applicable for the photodegradation of dye pollutants, particularly Congo red.

\section{Data Availability}

All the experimental results that we have obtained for this study were presented in this manuscript, and their discussion is supported by the references cited. I have no other data.

\section{Conflicts of Interest}

The authors declare that there are no conflicts of interest regarding the publication of this article.

\section{Acknowledgments}

The authors would like to thank the Environmental Pollution Chair at Princess Nourah Bint Abdulrahman University for financial support.

\section{References}

[1] G. Alagumuthu and T. Anantha kumar, "Structural, optical and photocatalytic activity of polymer capped zinc selenide nanoparticles," International Research Journal of Engineering and Technology, vol. 3, no. 11, pp. 1022-1028, 2016.

[2] N. Pandey, S. K. Shukla, and N. B. Singh, "Water purification by polymer nanocomposites: an overview," Nanocomposites, vol. 3, no. 2, pp. 47-66, 2017.

[3] N. Soltani, S. Elias, M. Z. Hussein et al., "Visible light-induced degradation of methylene blue in the presence of photocatalytic ZnS and CdS nanoparticles," International Journal of Molecular Science, vol. 13, no. 12, pp. 12242-12258, 2012.

[4] C. L. Torres-Martínez, R. Kho, O. I. Mian, and R. K. Mehra, "Efficient photocatalytic degradation of environmental pollutants with mass-produced ZnS nanocrystals," Journal of Colloid and Interface Science, vol. 240, no. 2, pp. 525-532, 2001.

[5] H. R. Pouretedal, A. Norozi, M. H. Keshavarz, and A. Semnani, "Nanoparticles of zinc sulfide doped with 
manganese, nickel and copper as nanophotocatalyst in the degradation of organic dyes," Journal of Hazardous Materials, vol. 162, no. 2-3, pp. 674-681, 2009.

[6] A. Di Mauro, M. Cantarella, G. Nicotra et al., "Novel synthesis of $\mathrm{ZnO} / \mathrm{PMMA}$ nanocomposites for photocatalytic applications," Scientific Reports, vol. 7, no. 1, pp. 1-12, 2017.

[7] F. Sayilkan and F. B. Emre, "Characterization and photocatalytic properties of $\mathrm{TiO}_{2}$ /chitosan nanocomposites synthesized by hydrothermal process," Turkish Journal of Chemistry, vol. 40, pp. 28-37, 2016.

[8] R. Rajendran, K. Varadharajan, V. Jayaraman, B. Singaram, and J. Jeyaram, "Photocatalytic degradation of metronidazole and methylene blue by PVA-assisted $\mathrm{Bi}_{2} \mathrm{WO}_{6}-\mathrm{CdS}$ nanocomposite film under visible light irradiation," Applied Nanoscience, vol. 8, no. 1-2, pp. 61-78, 2018.

[9] H. Zhu, R. Jiang, L. Xiao et al., "Photocatalytic decolorization and degradation of Congo red on innovative crosslinked chitosan/nano-CdS composite catalyst under visible light irradiation," Journal of Hazardous Materials, vol. 169, no. 1-3, pp. 933-940, 2009.

[10] R. S. Lankone, J. Wang, J. F. Ranville, and D. H. Fairbrother, "Photodegradation of polymer-CNT nanocomposites: effect of CNT loading and CNT release characteristics," Environmental Science: Nano, vol. 4, no. 4, pp. 967-982, 2017.

[11] Y. Jia, T. Wu, Z. Ding, and X. Li, "Preparation and characterization of carbon nanotubes/chitosancomposite foam with enhanced elastic property," Carbohydrate Polymers, vol. 136, pp. 1288-1296, 2016.

[12] R. Rajesh, A. Hariharasubramanian, N. Senthilkumar, and Y. Dominic Ravichandran, "A biocompatible and load bearing composite of multi walled carbon nanotubes chitosan and natural hydroxyapatite derived from the chicken bones wasted in the slaughter houses," International Journal of Pharmacy and Pharmaceutical Sciences, vol. 4, no. 4, pp. 716-720, 2012.

[13] O. W. Guirguis and M. T. H. Moselhey, "Thermal and structural studies of poly(vinyl alcohol) and hydroxypropyl cellulose blends," Natural Science, vol. 4, no. 1, pp. 57-67, 2012.

[14] H. Patel and R. T. Vashi, "Removal of Congo Red dye from its aqueous solution using natural coagulants," Journal of Saudi Chemical Society, vol. 16, no. 2, pp. 131-136, 2012.

[15] J. Li, S. Liu, Y. He, and J. Wang, "Adsorption and degradation of the cationic dyes over Co doped amorphous mesoporous titania-silica catalyst under UV and visible light irradiation," Microporous Mesoporous Material, vol. 115, no. 3, pp. 416425, 2008.

[16] F. Amri, S. Husseinsyah, and K. Hussin, "Effect of sodium dodecyl sulfate on mechanical and thermal properties of polypropylene/chitosan composites," Journal of Thermoplastic Composite Materials, vol. 26, no. 7, pp. 878-892, 2013.

[17] S. Chandra Dey, M. Al-Amin, T. U. Rashid et al., "Preparation, characterization and performance evaluation of chitosan as an adsorbent for remazol red," International Journal of Latest Research in Engineering and Technology, vol. 2, no. 2, pp. 52-62, 2016.

[18] P. Monvisade and P. Siriphannon, "Chitosan intercalated montmorillonite: preparation, characterization and cationic dye adsorption," Applied Clay Science, vol. 42, no. 3-4, pp. 427-431, 2009.

[19] L. Miao and Y.-J. Wu, "Preparation and characterisation of food-grade chitosan from housefly larvae," Czech Journal of Food Sciences, vol. 29, no. 6, pp. 616-623, 2011.
[20] J. Ghabboun, A. Ghaleb, M. Faroun et al., "Mapping of embedded functionalized carbon nanotubes in poly(vinyl alcohol)/nanotube composite using electrostatic force microscopy," International Journal of Polymer Analysis and Characterization, vol. 17, no. 4, pp. 268-277, 2012.

[21] T. Ungár, J. Gubicza, G. Ribárik, T. Cristian Pantea, and W. Zerda, "Microstructure of carbon blacks determined by X-ray diffraction profile analysis," Carbon, vol. 40, no. 6, pp. 929-937, 2002.

[22] Z. Abdeen and S. G. Mohammad, "Study of the adsorption efficiency of an eco-friendly carbohydrate polymer for contaminated aqueous solution by organophosphorus pesticide," Open Journal of Organic Polymer Materials, vol. 4, no. 1, pp. 16-28, 2014.

[23] N. A. Abdelwahab and A. M. Ghoneim, "Photocatalytic activity of $\mathrm{ZnO}$ coated magnetic crosslinked chitosan/polyvinyl alcohol microspheres," Materials Science and Engineering B, vol. 228, pp. 7-17, 2018.

[24] M. Thomas, G. Ahmad Naikoo, M. U. D. Sheikh, M. Bano, and F. Khan, "Effective photocatalytic degradation of Congo red dye using alginate/carboxymethyl cellulose/ $\mathrm{TiO}_{2}$ nanocomposite hydrogel under direct sunlight irradiation," Journal of Photochemistry and Photobiology A: Chemistry, vol. 327, pp. 33-43, 2016.

[25] M. G. Alalm and T. Ahmed, "Solar photocatalytic degradation of phenol in aqueous solutions using titanium dioxide," International Journal of Environmental and Ecological Engineering, vol. 8, no. 2, pp. 136-139, 2014.

[26] A. Franco, M. C. Neves, M. M. L. R. Carrott, M. H. Mendonça, M. I. Pereira, and O. C. Monteiro, "Photocatalytic decolorization of methylene blue in the presence of $\mathrm{TiO}_{2} / \mathrm{ZnS}$ nanocomposites," Journal of Hazardous Materials, vol. 161, no. 1, pp. 545-550, 2009.

[27] E. S. Elmolla and M. Chaudhuri, "Degradation of amoxicillin, ampicillin and cloxacillin antibiotics in aqueous solution by the UV/ZnO photocatalytic process," Journal of Hazardous Materials, vol. 173, no. 1-3, pp. 445-449, 2010.

[28] J. Blanco, S. Malato, P. Fernández et al., "Compound parabolic concentrator technology development to commercial solar detoxification applications," Solar Energy, vol. 67, no. 4-6, pp. 317-330, 1999.

[29] A. Nithya, K. Jothivenkatachalam, S. Prabhu, and K. Jeganathan, "Chitosan based nanocomposite materials as photocatalyst - a review," Materials Science Forum, vol. 781, pp. 79-94, 2014.

[30] S. A. Umoren, U. J. Etim, and A. U. Israel, "Adsorption of methylene blue from industrial effluent using poly (vinyl alcohol)," Journal of Materials and Environmental Science, vol. 4, no. 1, pp. 75-86, 2013. 

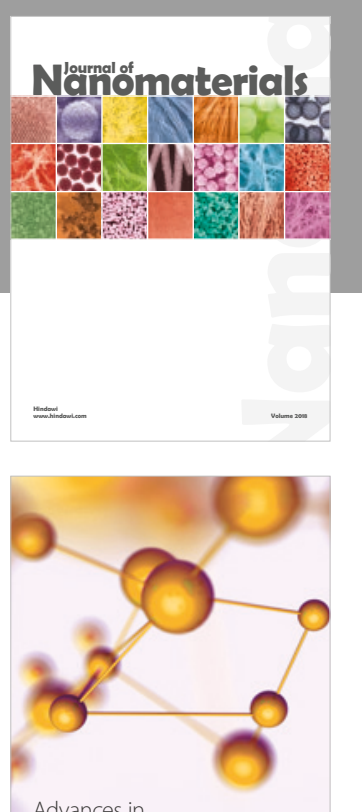

Physical Chemistry
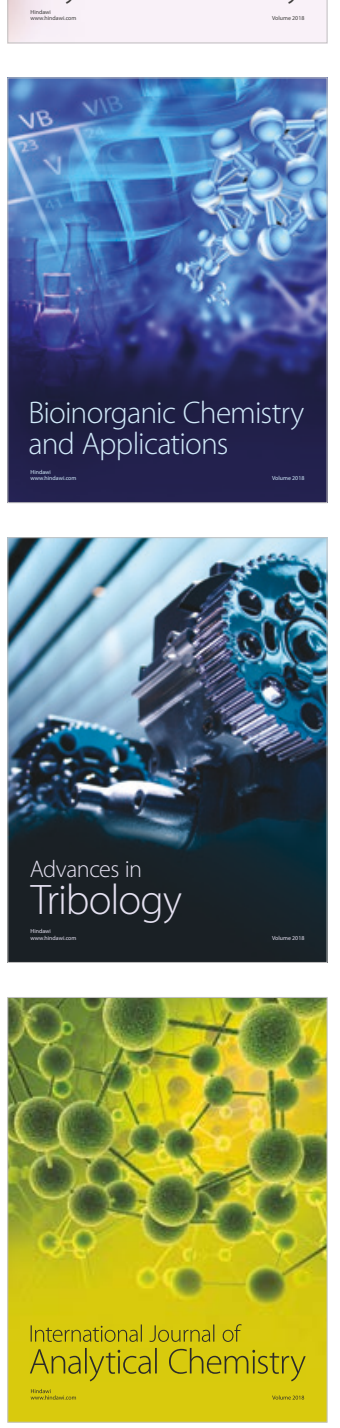

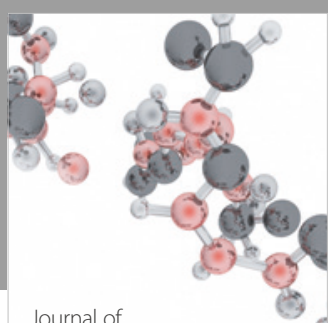

Analytical Methods

in Chemistry

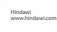

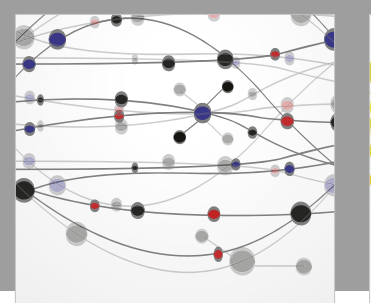

The Scientific World Journal



Journal of

Applied Chemistry
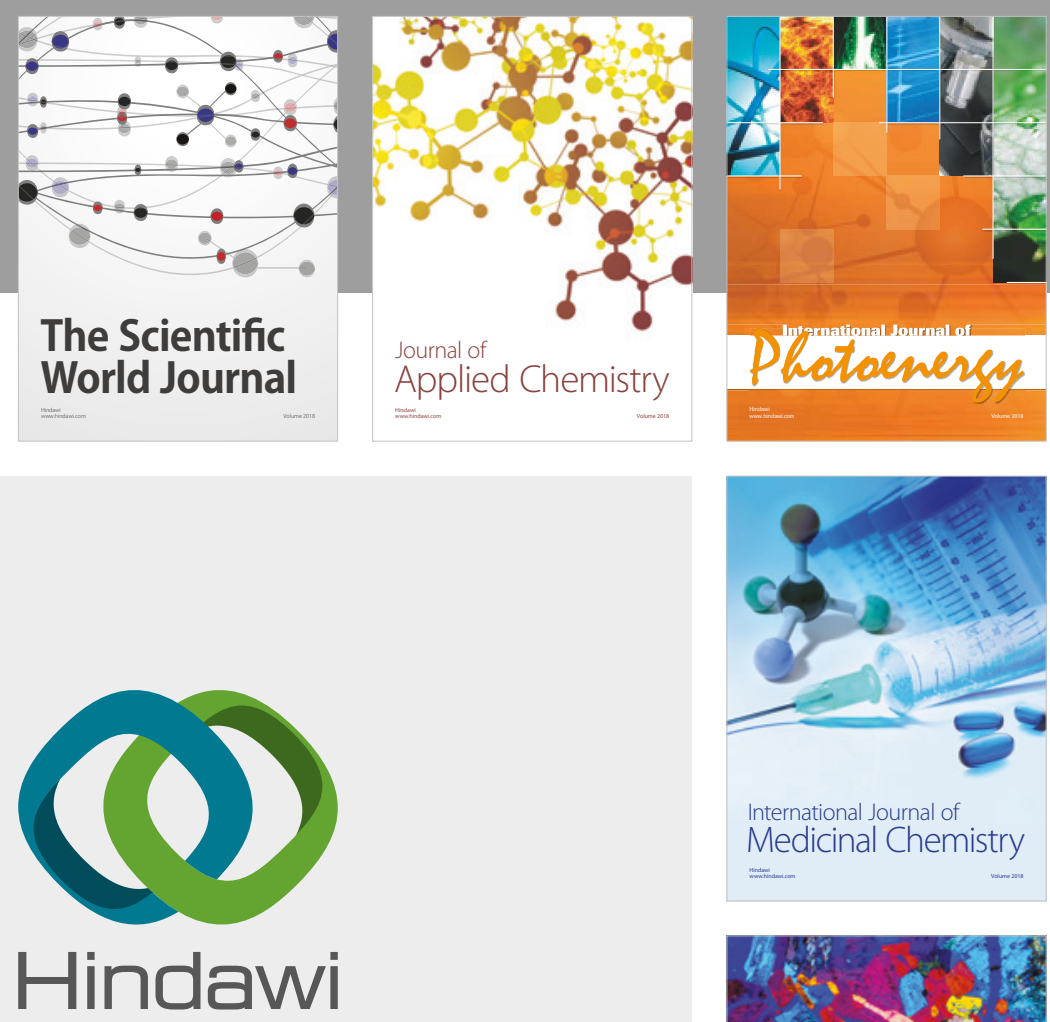

Submit your manuscripts at

www.hindawi.com

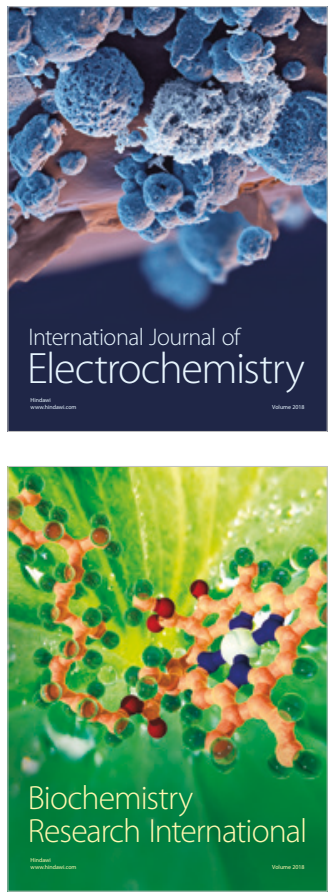